\title{
Estrategia de Competitividad y Emprendimiento, una Revisión de la Literatura
}

\author{
Competitive Strategy and Entrepreneurship, a literary revision
}

\begin{abstract}
Angélica María Jiménez Coronado
Ingeniero de Sistemas - Universidad Autónoma del Caribe, Especialista en Redes de Computadores - Universidad del Norte, Magister en Administración de Empresas - Universidad del Norte y Doctorante en Administración - Escuela de Negocios, Universidad del Norte: Becaria por el Programa de Apoyo Financiero. Docente Tiempo Completo del Programa de Administración de Empresas Universidad del Atlántico.

Correo: angelicajimenez@mail.uniatlantico.edu.co
\end{abstract}




\section{Resumen}

A ctualmente, la globalización conecta sas, haciéndolas más competitivas en el tiempo. En efecto, los analistas de las estrategias y la competitividad se han vuelto instrumentos de planeación indispensables para aumentar la participación del mercado. Este paper construye una revisión literaria de las teorías epistemológicas de competitividad, estrategia, emprendimiento y alianzas estratégicas capaces de incorporarse en los modelos de desarrollo económico del país por medio del contexto empresarial. Se recomienda, mediante la metodología, se incorporen los modelos para fomentar la creación de empresas y construir capital social. Seguidamente, muestra el impacto de la pertinencia de las inversiones a nivel macro y micro para competir con más eficacia. Por último, se hace un balance del impacto y la pertinencia de los temas tratados y los vacíos existentes, con el fin de orientar futuras investigaciones.
Palabras clave:

Emprendimiento, Competitividad, Estrategia, Alianzas, Modelo de Competitividad.

\section{Abstract}

C urrently, globalization connects with great vitality companies managing to be competitive over time, in fact the analysts of competitiveness and strategies have become indispensable planning instruments that seek to increase market share. This Paper builds a literary review of the epistemological theories of competitiveness, strategy, entrepreneuKeywords:

Entrepreneurship, Competitiveness, Strategy, Alliances, Competitiveness Model rship and strategic alliances capable of being incorporated in the models of the country's economic development through the business context. It is recommended through the methodology to incorporate models for promoting the creation of new firms and building social capital. Then, it shows the impact of the relevance of investments at micro and macro level in order to compete more effectively. Finally, an assessment of the impact and relevance of existing issues and gaps that may be able to guide future research work was done here. 


\section{Introducción}

A ctualmente, las empresas compiten por los mercados con gran rivalidad por tener los mejores productos en épocas de gran demanda. Es por esto que los analistas de las estrategias y la competitividad han pasado a ser instrumentos de planeación indispensables para aumentar la participación del mercado. Según Porter, la definición de una estrategia competitiva consiste en desarrollar una amplia fórmula de cómo la empresa va a competir, cuáles deben ser sus objetivos y qué políticas son necesarias para alcanzar tales objetivos.

Asimismo, ha construido un esquema conceptual que explica la prosperidad a partir de tres factores genéricos: dotación de recursos, competitividad macroeconómica y competitividad microeconómica (ver Figura 1).

Es por esto que se puede afirmar que el emprendimiento es hoy de gran interés, dado que logra contribuir al desarrollo de los países de origen, estimular la innovación, fomentar la creación de empresas pequeñas y medianas y generar empleo, además de crear capital social a través de las fronteras.

Los factores que influyen en el emprendimiento constituyen el ingrediente principal que puede llevar a una empresa al éxito $\mathrm{y}$, por tanto, a ser competitiva. Para los emprendedores y las PYMES con negocios en Internet, las alianzas estratégicas son una poderosa herramienta de marketing y una excelente oportunidad de colaboración para competir, ya que estas buscan siempre el beneficio mutuo.

Conocidas también como "joint ventures", se trata de un acuerdo entre empresas mediante el cual unen fuerzas para conse-

\section{Fuente: Moving to a new global competitiveness index. The global competitiveness report [1].}

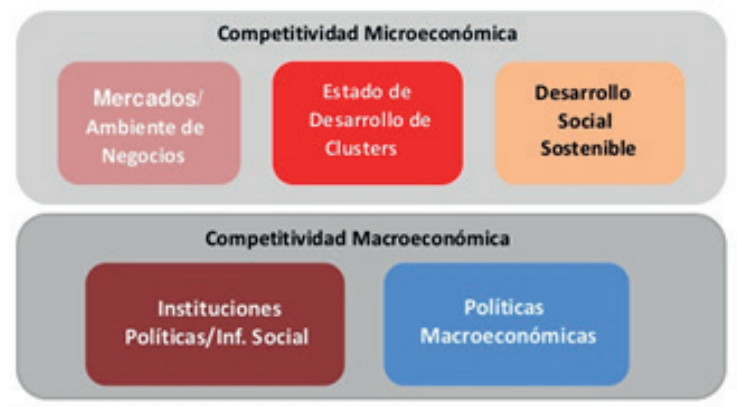

DOTACIONES

guir un objetivo estratégico común. El objetivo estratégico de esta unión de fuerzas puede ser: superar barreras comerciales en nuevos mercado, desarrollar productos o servicios y acceder a mercados extranjeros que requieren de importantes inversiones y de un conocimiento del mercado (know-how) para desarrollar marca país, lo que permite ingresar a zonas geográficas específicas.

Las razones mencionadas anteriormente motivan la realización de este artículo, el cual revisa la literatura que analiza la relación entre estrategia de competitividad, emprendimiento y alianzas como herramientas de valor agregado. De esta manera, se considera el emprendimiento como el mecanismo que articula la innovación para incrementar la productividad, a condición de disponer de los recursos humanos y tecnológicos adecuados; y la estrategia de competitividad como el fenómeno 
directamente relacionado con el desempeño y eficiencia técnica de las empresas y las alianzas que contribuyen al desarrollo económico-social.

En la primera sección de este artículo se consideran algunos aspectos teóricos de la Estrategia y Competitividad, el Emprendimiento y las Alianzas, como su definición, sus causas y teorías que las explican. La segunda sección expone la evidencia empírica sobre la relación entre Estrategia de Competitividad y Emprendimiento con un entorno de alianzas específicas. En la tercera sección se comentan los aspectos metodológicos que han debido considerar las investigaciones empíricas. Por último, se hace un balance del impacto y la pertinencia de los temas tratados y los vacíos existentes, lo cual puede orientar futuras investigaciones.

\section{Abordaje teórico de competitividad y estrategia.}

Competitividad

El término competitividad es muy utilizado en el contexto empresarial, político y socioeconómico en general. A ello se debe la ampliación del marco de referencia de nuestros agentes económicos que han pasado de una actitud autoprotectora a un planteamiento más abierto, expansivo y proactivo. La competitividad tiene incidencia en la forma de plantear y desarrollar cualquier iniciativa de negocios, lo que está provocando una evolución en el modelo de empresa y empresario. La ventaja comparativa de una empresa estaría en la habilidad, recursos, conocimientos y atributos, etc. de los que dispone, los mismos que carecen sus competidores o que tienen en menor medida, y que hace posible la obtención de unos rendimientos superiores a los de aquellos. Por otra parte, el concepto de competitividad nos hace pensar en la idea de "excelencia", es decir, con características de eficiencia y eficacia de la organización.

Los mercados se han vuelto altamente competitivos y debido a su rápido crecimiento están en constante cambio y pasan de ser simples a complejos, de estables a dinámicos [2], no importando el tamaño de las empresas que lo conformen.

Al ser la competitividad el objetivo de las organizaciones, en los últimos años se han generado diversos modelos propuestos por expertos con el fin de elevarla.

De forma conceptual, la competitividad se concibe como un fenómeno empresarial o patrón organizativo de la sociedad, relacionándose con su comportamiento en el mercado en el que opera [3] y manteniendo e incrementando su participación basada en nuevas estrategias [4]. En la mayoría de los casos depende de la productividad, rentabilidad, posición competitiva y participación en el mercado interno y externo.

\section{Competitividad Empresarial}

Es indudable que al referirse a la competitividad de una firma se están tomando en cuenta mercados en los cuales los productores tienen capacidad de controlar el proceso de fijación de precios, a diferencia de los mercados de libre competencia, en los que los precios son establecidos por el mercado. En mercados oligopólicos y donde la competencia se hace no solo por precios sino por diferenciación de productos, las firmas pueden aumentar su participación en el mercado a través del lanzamiento de nuevos productos y la puesta 
en práctica de nuevos procesos de producción, además de las prácticas habituales de propaganda y publicidad [1].

Para poder lograr una mayor competitividad, la firma puede recurrir a diversos instrumentos como una mejor gestión financiera [5], disponer de activos tales como una mayor capacidad innovadora en materia de nuevos productos y procesos de producción, lograr un aumento mayor al de sus competidores en productividad de la mano de obra y del capital, reducir sus costos a través de una mayor integración vertical (reduciendo los costos de transacción) o, en otros casos, por medio de una descentralización de la producción en el país (subcontratación) o a través de inversiones o licencias en el exterior, etcétera. Desde los inicios, los mismos factores que ayudarían a explicar la competitividad de una firma en su propio mercado, es decir, a nivel nacional, servirían para explicar la Competitividad Internacional de la firma frente a las importaciones y/o con sus exportaciones en mercados externos. Sin embargo, el tipo de cambio y la configuración de la política, tanto en el mercado interno como externo, hacen intervenir cuestiones macroeconómicas en la determinación de la Competitividad Internacional.

Seguidamente, el camino que la firma utilice para su expansión internacional también debe ser considerado con el fin de evaluar su Competitividad Internacional. La competitividad de las empresas depende de factores en tres niveles: el primer nivel es la competitividad del país, que incluye variables como la estabilidad macroeconómica, la apertura y acceso a mercados internacionales o la complejidad de la regulación para el sector empresarial; el segundo nivel se refiere a la infraestructura regional; en tercer nivel, la competitividad de las empresas tiene que ver con lo que ocurre dentro de la propia empresa [6]. La competitividad empresarial se deriva de la ventaja competitiva que tiene una empresa a través de sus métodos de producción y de organización (reflejados en precio y en calidad del producto final) con relación a los de sus rivales en un mercado específico. $[7,8]$ han señalado que las que compiten son las empresas no las naciones, es decir, a un país lo hacen competitivo las empresas competitivas que hay en éste.

\section{Modelo de Competitividad}

El ámbito económico, político y social de un país sirve para hacer un diagnóstico del papel del Estado en la economía, ya que es el ente regulador de su propia actividad. Valencia [9] muestra en la Tabla 1 los modelos de competitividad que son capaces de generar las ventajas competitivas requeridas para una inserción exitosa en la dinámica de la economía mundial. En un entorno de profundos rezagos estructurales y de numerosos problemas de competitividad, la carencia de mecanismos de fomento ha sido una de las principales deficiencias de la estrategia modernizadora. 


\begin{tabular}{|c|c|}
\hline Modelo & Descripción \\
\hline $\begin{array}{l}\text { Informe Global de } \\
\text { Competitividad (IGC) }\end{array}$ & $\begin{array}{l}\text { Desarrollado por el World Economic Forum (WEF). La } \\
\text { primera publicación de este índice de competitividad se } \\
\text { da en el año de } 1979 \text { con el índice de Competitividad } \\
\text { Global, donde el desarrollo de los países se medía a } \\
\text { través d e instrumentos e specíficos, " los índices", } \\
\text { utilizando } 5 \text { variables. C ontempla nueve p ilares } \\
\text { fundamentales: i nstituciones, infraestructura, } \\
\text { macroeconomía, s alud y e ducación, capacitación } \\
\text { laboral, eficiencia en el mercado, sofisticación de los } \\
\text { negocios, innovación y r apidez e n la a dopción de } \\
\text { nuevas tecnologías }\end{array}$ \\
\hline $\begin{array}{l}\text { Enfoque del World } \\
\text { Competitiveness Center } \\
\text { (WCC) del Institute for } \\
\text { Management and } \\
\text { Development (IMD) }\end{array}$ & $\begin{array}{l}\text { El W orld C ompetitiveness R eport es c onsiderado e I } \\
\text { reporte de competitividad más completo y detallado. Es } \\
\text { publicado desde } 1989 \text { e } n \text { el a nuario m undial d e } \\
\text { competitividad y e } \mathrm{s} \text { elaborado por el I nstituto } \\
\text { Internacional para el Desarrollo de la Administración. } \\
\text { Este í ndice divide e I ambiente } n \text { acional en c uatro } \\
\text { factores principales (desempeño económico, eficiencia } \\
\text { del gobierno, e ficiencia en I os n egocios e } \\
\text { infraestructura), que a su v ez s e dividen en c inco } \\
\text { subfactores que destacan d iferentes facetas de I a } \\
\text { competitividad. A cada subfactor se le ha asignado un } \\
\text { peso r elativo del } 5 \% \text { p ara la c onsolidación d e los } \\
\text { resultados, teniendo en cuenta que este porcentaje es } \\
\text { independiente del número de criterios que cada uno } \\
\text { tiene para hacer la calificación. }\end{array}$ \\
\hline
\end{tabular}

Tabla 1. Modelos de competitividad 
Fuente: Variables e Competitiveness Center Índices de (WCC) del Institute for Competitividad de las Empresas Exportadoras, utilizando el PLS

[9].

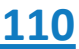

Management and

Development (IMD)

Índice de

Competitividad IMCO
Modelo: Heritage Foundation
El World Competitiveness Report es considerado el reporte de competitividad más completo y detallado. Es publicado desde 1989 en el anuario mundial de competitividad y es elaborado por el Instituto Internacional para el Desarrollo de la Administración. Este índice divide el ambiente nacional en cuatro factores principales (desempeño económico, eficiencia del gobierno, eficiencia en los negocios e infraestructura), que a su vez se dividen en cinco subfactores que destacan diferentes facetas de la competitividad. A cada subfactor se le ha asignado un peso relativo del $5 \%$ para la consolidación de los resultados, teniendo en cuenta que este porcentaje es independiente del número de criterios que cada uno tiene para hacer la calificación.

El Instituto Mexicano para la Competitividad ha manejado diferentes mediciones. Existen dos índices: el primero es el índice de competitividad internacional y el segundo es el índice de competitividad estatal. En el primer índice se manejan las siguientes variables: derecho, medio ambiente, sociedad, economía, política, factores precursores, gobierno, relaciones internacionales, sofisticación e innovación. En el segundo índice se manejan las siguientes variables: sistema de derecho confiable y objetivo; manejo sustentable del medio ambiente; sociedad incluyente, preparada y sana; economía estable y dinámica; sistema político estable y funcional; mercado de factores eficientes; sectores precursores de clase mundial; gobiernos eficientes y eficaces; aprovechamiento de las relaciones internacionales; sectores económicos en vigorosa competencia. De ellas surgen 118 indicadores en total.

Este organismo publica el "índice de libertad económica", el cual está integrado por datos organizados en 10 categorías, incluidas la tasa impositiva, la política monetaria, la inflación, los derechos de propiedad y el marco regulatorio. Por "libertad económica" se entiende la falta de coerción o coacción del Estado en la producción, la distribución o el consumo de bienes y servicios. El estudio abarca 161 países y sus resultados muestran, entre otras cosas, que los "países con los niveles más altos de libertad 
De acuerdo con Porter, el Diamante de la Competitividad cuenta con cuatro factores determinantes de la ventaja competitiva:

1. Las condiciones de los factores. Incluyen los factores de producción (mano de obra calificada, infraestructura, financiamiento).

2. Condiciones de la demanda. Se refiere al grado de complejidad de la demanda en el mercado para los bienes o servicios producidos por una industria determinada.
3. Industrias asociadas y de apoyo. La presencia de proveedores y de industrias conexas que sean internacionalmente competitivas aumenta la influencia de aquellas que las rodean.

4. Estrategia, estructura y rivalidad de la empresa. Las condiciones que impone la manera como las firmas son creadas, organizadas y dirigidas, así como la naturaleza de la competencia doméstica. Una fuerte competencia interior nutre el éxito al exterior (ver Figura 2).

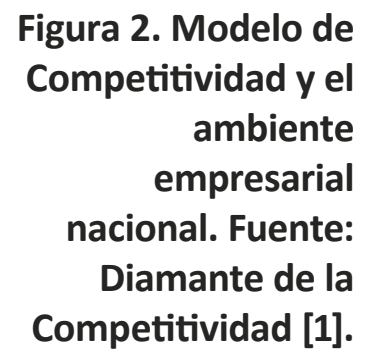

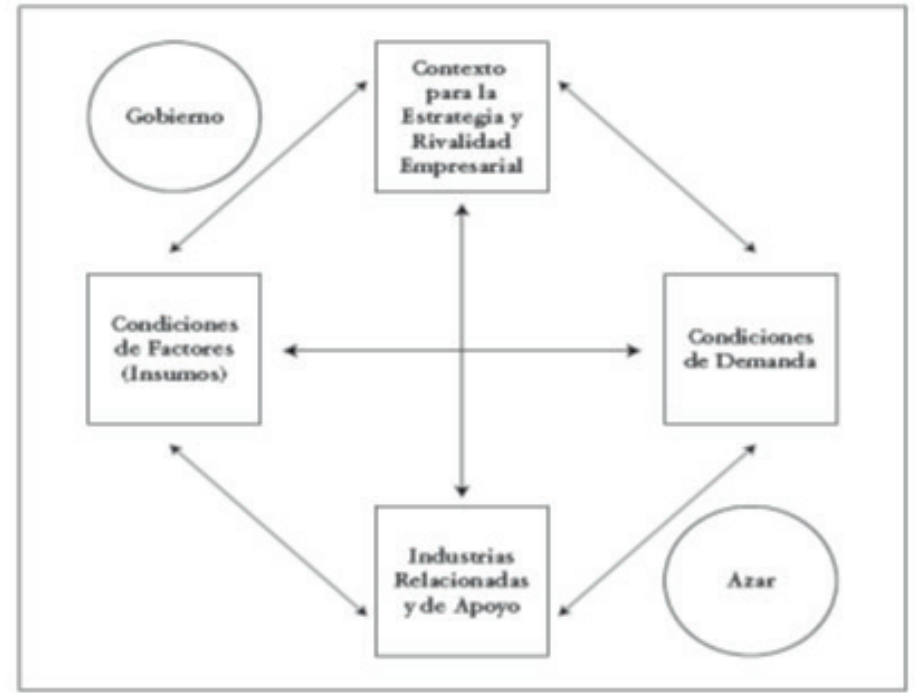

En Colombia, el modelo de competitividad lo lidera el Sistema Nacional de Competitividad, Ciencia Tecnología e Innovacion - SNCCTI, el cual está garantizado por medio del concepto de Competitividad de una nación, según el documento CONPES 3934 de 2009, el cual se define como:
“El grado en el que un país puede producir bienes y servicios capaces de competir exitosamente en mercados globalizados y a la vez mejorar las condiciones de ingreso y calidad de vida de su población. La competitividad es el resultado de la interacción de múltiples factores relacionados con las condiciones que enfrenta la actividad empresarial y que condicionan su desempeño, tales como infraestructura, recursos humanos, ciencia y tecnología, instituciones, entorno macroeconómico y productividad". 
A nivel internacional, los indicadores de competitividad más conocidos son el Índice de Competitividad Global (Index Global Competitiviness, ICG), realizado por el Foro Económico Mundial (World Economic Forum, WEF); el Anuario Mundial de Competitividad, elaborado por el Instituto Internacional para el Desarrollo Gerencial (International Institute for Management Development, IIMD) y el Índice de Facilidad para hacer Negocios (Doing Business), desarrollado por el Grupo del Banco Mundial (World Bank Group).
En términos de innovación, el Global Innovation Index (Índice Global de Innovación), realizado de forma conjunta por la Organización Mundial de la Propiedad Intelectual (OMPI), la Universidad Corne11 y la Escuela de Negocios INSEAD; se ha consolidado como un índice de referencia mundial para evaluar la innovación en los países.

A continuación se observa el posicionamiento de Colombia ante los países del mundo en el tema de competitividad:

\section{Tabla 2. Posición Indicador de Competitividad del país Colombia ante los países del mundo.}

\section{2}

Fuente: Sistema Nacional de Competitividad, Ciencia

Tecnología e Innovación SNCCTI, 2016.

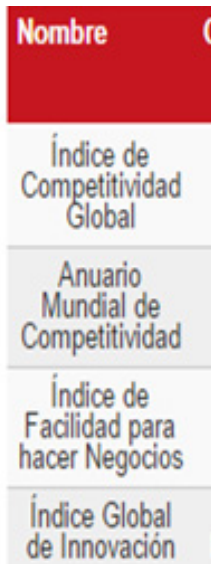

\section{Organización}

total de No. Posición Top 5
total de de
Paises Colombia

Foro Económico Mundial
Instituto Internacional para el Desarrollo Gerencial Grupo del Banco Mundial

Organización Mundial de la Propiedad de Innovación Intelectual (OMPI), la Universidad Cornell y la Escuela de Negocios INSEAD
$140 \quad 61 \quad$ Suiza Singapur Estados Unidos Alemania Paises Bajos.

\begin{tabular}{ccc}
61 & 51 & $\begin{array}{c}\text { Estados Unidos, China - Hong } \\
\text { Kong Singapur, Suiza y Canadá }\end{array}$ \\
189 & 54 & $\begin{array}{c}\text { Singapur, Nueva Zelanda } \\
\text { Dingamarca, La República de } \\
\text { Corea y China - Hong Kong }\end{array}$ \\
\hline 141 & 67 & $\begin{array}{l}\text { Suiza, Reino Unido, Suecia, } \\
\text { Holanda y Estados Unidos }\end{array}$
\end{tabular}

A nivel nacional, se encuentra el Informe Nacional de Competitividad y el Índice Departamental de Competitividad, realizado por el Consejo Privado de Competitividad (CPC), organización privada sin ánimo de lucro, creada en el año 2006 por un grupo de empresarios y universidades que buscaban proponer e impulsar iniciativas y estrategias para mejorar significativamente la competitividad del país. El CPC actúa como articulador e interlocutor entre el sector público, el sector privado, la academia y otras organizaciones interesadas en la promoción de la competitividad y la productividad. Las variables de estudio de dicho informe son: educación, pensiones, ciencia, tecnología y educación, tic, sistema tributario, corrupción, política comercial, salud, mercado laboral y formalización, infraestructura, transporte y logística, financiamiento para el emprendimiento, justicia y energía.

Es necesario conocer que para el mejoramiento de la competitividad es necesario apoyar los proyectos de inversión nacional en: crecimiento en ciencia y tecnología, infraestructura y logística, educación, reducción y optimización de las tasas impositivas a las rentas corporativas $y$, finalmente, la reducción de la informalidad. Esto último por medio del aumento de los beneficios para la formalización. 


\section{La competitividad y la estrategia em- presarial}

La competitividad no es producto de una casualidad ni surge espontáneamente, se crea y se logra a través de un largo proceso de aprendizaje y negociación por parte de grupos colectivos representativos que configuran la dinámica de conducta organizativa, como los accionistas. El término competitividad es muy utilizado en los medios empresariales, políticos y socioeconómicos en general. A ello se debe la ampliación del marco de referencia de nuestros agentes económicos que han pasado de una actitud autoprotectora a un planteamiento más abierto, expansivo y proactivo.

La competitividad tiene incidencia en la forma de plantear y desarrollar cualquier iniciativa de negocios, lo que está provocando obviamente una evolución en el modelo de empresa y empresario.

La ventaja comparativa de una empresa estaría en la habilidad, recursos, conocimientos y atributos, etc. de los que dispone, los mismos que carecen sus competidores o tienen en menor medida, y que hace posible la obtención de rendimientos superiores a los de su competencia.

El uso de estos conceptos supone una continua orientación hacia el entorno y una actitud estratégica tanto de las empresas grandes como de las pequeñas, en las de reciente creación como en las maduras y, en general, en cualquier clase de organización. Por otra parte, el concepto de competitividad nos hace pensar en la idea de "excelencia", es decir, en características de eficiencia y eficacia de la organización.

\section{Estrategia}

Surge en China y después en la antigua Grecia, en el siglo IV a. de. C., en donde se circunscribe el origen etimológico de la expresión strategos o "general o jefe del ejército"; no muy alejada de la visión del siglo XXI.

Michael Porter [10] define la estrategia así: "La estrategia es la búsqueda de una posición favorable dentro de una industria, escenario fundamental donde se lleva a cabo la competencia. Su finalidad es establecer una posición más rentable y sustentable frente a las fuerzas que rigen la competencia en la industria; implica la utilización y profunda integración del poder económico, político, cultural, social, moral, espiritual y psicológico".

Lo anterior nos lleva a estudiar en profundidad cuatro ámbitos principales con sus correspondientes situaciones y acciones, de los que se derivan los caracteres que explican cada ámbito y son comunes en cada situación: 
Tabla 3. Concepto de estrategia: ámbitos de aplicación y caracteres comunes

Fuente: Teoría de la estrategia y la competitividad: estado del arte desde la perspectiva de Michael

E. Porter y su aplicación en Colombia [11].

\begin{tabular}{|l|}
\hline \multicolumn{1}{|l}{ Ámbito: guerra } \\
\hline Campo de batalla: Entorno (conjunto \\
de factores externos, no controlables \\
pero predecibles). \\
Situación de conflicto: Rivalidad. \\
Sistema de objetivos (en esencia \\
ganar) y conducta inteligente. \\
Recursos y capacidades. \\
\hline Ámbito: política \\
\hline Circunscripción administrativa. Entorno \\
(conjunto de factores externos, no \\
controlables pero predecibles). \\
Situación de rivalidad. Colusión de \\
intereses. Sistema de objetivos (en \\
esencia ganar y coaliciones) y conducta \\
inteligente. \\
Recursos y capacidades. $\square$
\end{tabular}

Ámbito: juegos

Campo de juego: Entorno (conjunto de factores externos, no controlables pero predecibles).

Situación de conflicto. Rivalidad. Sistema de objetivos (en esencia ganar) y conducta inteligente. Recursos y capacidades. Ámbito: negocios $\square$ Campo de actividad (mercado). Entorno (conjunto de variables externas, no controlables pero previsibles). Situación de rivalidad. Competencia. Sistema de objetivos (en esencia ganar y crecer) y conducta inteligente. Recursos y capacidades.

También podríamos llegar a conocer el concepto de estrategia de negocios: es la forma en la cual un single-business (negocio en particular) o una unidad de negocios individual del tamaño de una compañía compite en una industria en particular o en el mercado. La estrategia corporativa es la forma en la cual se administra una corporación de un conjunto de negocios.

Es importante identificar, desde la epistemología clásica, como se hace hincapié en los métodos racionales de planificación que dominan los textos. Alfred Chandler [12] afirma que la estrategia está determinada por el manejo de la información y tiene un valor determinante en la estructura de la empresa. "Si la estructura no sigue la estrategia, el resultado final es la ineficiencia". Seguidamente, Ansoff [13] identifica cuatro componentes de la estrategia que son: ámbito producto-mercado, un vector de crecimiento, ventajas compe- titivas y sinergia; exhibiendo un ámbito para el cual la empresa puede desarrollar su estrategia. Subsiguientemente, Alfred Sloan estableció que: "El objetivo estratégico de una empresa es obtener un rendimiento sobre los beneficios, y si en algún caso el rendimiento a largo plazo no es satisfactorio, el déficit debe ser corregido o la actividad abandonada". El último y quizás el más famoso precursor de esta perspectiva en la actualidad es Michael E. Porter [10], denominado el gurú de la estrategia y la competitividad por sus aportes en la utilización de modelos analíticos para determinar la posición competitiva en determinada industria.

Seguidamente, se quieren conocer las características básicas de la estrategia en el enfoque clásico: la defensa del análisis racional, la separación entre concepción y ejecución y el compromiso con la maximización de los beneficios. Se concluye que la planificación puede adaptarse y antici- 
parse a los cambios del mercado y que el mejor modo de diseñar una estrategia es a través del análisis racional alejado de la interferencia de la competencia.

Siguiendo con los enfoques, a continuación se describe el evolucionista, el cual parte de la influencia del concepto de "selección natural" en la economía de las organizaciones, caracterizado, principalmente, por la desconfianza en los top management. Este enfoque espera siempre que los mercados garanticen unos beneficios máximos, y considera que la competencia no consiste en un cálculo independiente, sino en la constante lucha por sobrevivir. Esta teoría ofrece una paradoja cruel: "La naturaleza dinámica, hostil y competitiva de los mercados supone que no es posible planificar la supervivencia a largo plazo; en consecuencia, únicamente sobrevivirán empresas que den con estrategias que maximicen los beneficios". Desde esta perspectiva, es el mercado, y no los directivos, el que determina las decisiones más importantes a tomar, es decir, las buenas estrategias surgen únicamente como producto de la selección natural y lo único que pueden hacer los directivos es estar preparados para las exigencias del entorno. Bruce Henderson, fundador del prestigioso Boston Consulting Group, definió la estrategia de tal manera que limita dos tendencias: la competencia estratégica y la competencia natural (ver Tabla 4).

\begin{tabular}{|c|c|c|}
\hline Tabla 4. & Competencia estratégica & Competencia natural \\
\hline $\begin{array}{r}\text { Fuente: Olas } \\
\text { paradigmáticas } \\
\text { de la estrategia: } \\
\text { enfoque } \\
\text { hermenéutico } \\
{[14] .}\end{array}$ & $\begin{array}{l}\text { La capacidad de entender la interacción } \\
\text { entre competidores c omo un s istema } \\
\text { dinámico completo. } \\
\text { La c apacidad d e predecir I as } \\
\text { consecuencias de u na i ntervención } \\
\text { concreta. } \\
\text { La d isponibilidad de r ecursos no } \\
\text { comprometidos que pueden d edicarse } \\
\text { actualmente a diferentes usos y fines. } \\
\text { La b uena d isposición p ara actuar } \\
\text { decididamente y comprometer esos } \\
\text { recursos son características. }\end{array}$ & $\begin{array}{l}\text { Es t remendamente o portunista e n las } \\
\text { interacciones de c ada momento. } \\
\text { Extremadamente c onservadora en e I } \\
\text { cambio de comportamiento. } \\
\text { Evolutiva y procede por tanteos, p or } \\
\text { pequeños i ncrementos y c on p oco } \\
\text { riesgo. }\end{array}$ \\
\hline
\end{tabular}

El enfoque clásico y evolucionista consideran la maximización de beneficios como el resultado lógico de la aplicación de una estrategia; el enfoque sistémico y el procesual tienen una visión más plural, aceptan que pueden existir otros resultados aparte de los beneficios. Cabe anotar la importancia de construir un proceso capaz de dar valor agregado a las estrategias evolucionistas con el fin de pronosticar los resultados óptimos que se quieren alcanzar (ver Figura 3). 
Figura 3. ¿Qué es la estrategia? ¿Realmente importa? Thomson.

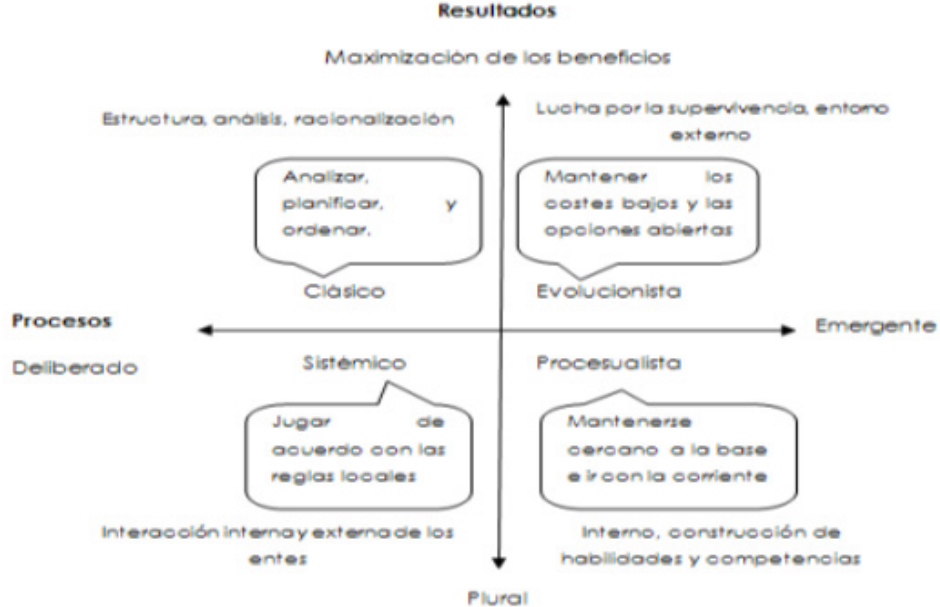

Por lo tanto, al identificar el enfoque procesualista podemos decir que es de carácter evolutivo como el enfoque anterior, y que comparte con este el escepticismo sobre la utilidad de la formulación racional de la estrategia, pues hace énfasis en la naturaleza complicada e imperfecta de los seres humanos $\mathrm{y}$, por tanto, acomoda la estrategia de un modo pragmático a los erráticos procesos tanto de las organizaciones como de los mercados. De la misma manera, los procesos tanto de las organizaciones como de los mercados suelen no ser lo suficientemente perfectos como para cumplir con los estrictos requisitos de la

teórica clásica o con la despiadada lucha por la supervivencia de los evolucionistas. Indudablemente, es necesario construir, desde los sectores, una panorámica para conocer de forma macro y meso las políticas gubernamentales a nivel regional. Lo proponen Altenburg et al. [15] y Lombana [16], y explican cómo el nivel es el vínculo analítico entre lo macro y lo micro. El parámetro regional, que hasta ahora no se ha incluido, puede contextualizarse en el modelo sistémico de la competitividad que Altenburg et al. definieron en 1998 en la Figura 4:

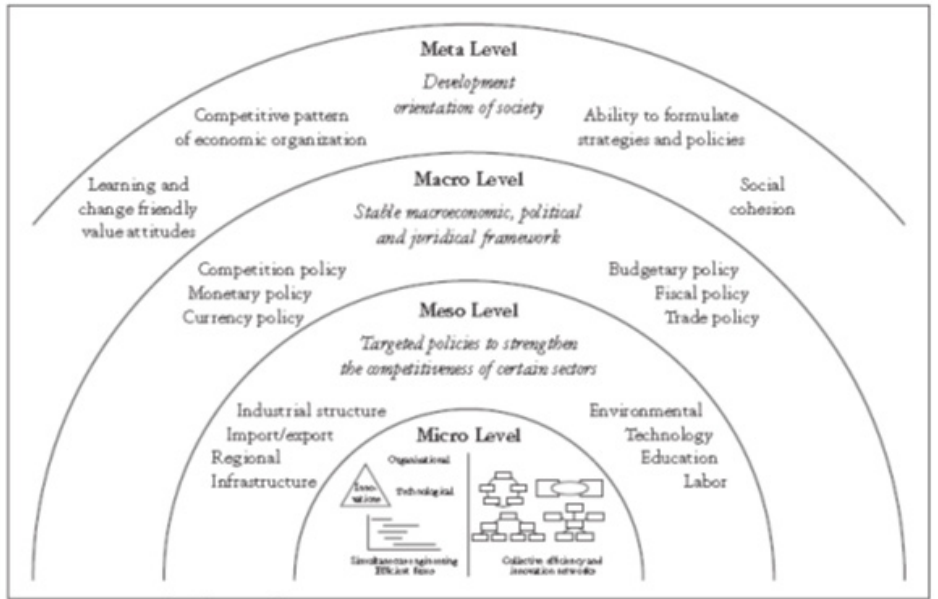

Figura 4.

Competitividad sistémica. Fuente: [15].

Las actividades desde este punto son vitales para el desarrollo de la competitividad en la cohesión social que estos puedan crear, con el fin de ejercer la gobernabilidad desde la gestión de los recursos económicos y sociales para el desarrollo del país. 


\section{Teorías del emprendimiento social y las alianzas estratégicas: fenómeno en el desarrollo económico.}

Desde la mirada del trabajo seminal de Cantillón [17] hasta el trabajo realizado por Shane y Venkataraman [18], se ha relacionado el emprendimiento con el descubrimiento de oportunidades rentables. De hecho, estos autores toman la definición de oportunidad de Casson [19] y declaran que "las oportunidades de emprendimiento son aquellas situaciones en las que nuevos bienes, servicios, materias primas y métodos de organización pueden ser vendidos e introducidos a un precio mayor que sus costos de producción" [18]. "El emprendedor es entonces quien descubre, evalúa y explota oportunidades rentables, tomando en cuenta el riesgo, alerta a las oportunidades y necesidad por la innovación. ¿Entonces dónde encaja el aspecto social al interior de este marco conceptual para el emprendimiento?". La mayoría de la población excluida de la economía son pobres en general: ¿dónde se genera valor? [20]. Este cuestionamiento facilita el dar inicio a una discusión que concluya en la presentación de una definición de emprendimiento social y las alianzas estratégicas.

Emprendimiento Social

El emprendimiento constituye un fenómeno cultural que encierra conductas, valores, creencias y modos de actuación, con la intencionalidad de generar bienestar social en una comunidad. A su vez, la cultura constituye una variable importante, tanto para el proceso de desarrollo de la idea emprendedora como para la acción o puesta en marcha [21].

Desde un enfoque filosófico, expresado en el pensamiento de Husser, explicitar la correlación entre el hombre, su realidad y el mundo de las cosas, es decir, la estructura de la experiencia humana, es lo que se ha llamado fenomenología.

Desde la mirada de la fenomenología, el lugar donde se desarrolla la cultura se denomina el mundo social o campo de acción que se organiza alrededor de la persona, según sus planes y significaciones, sin dejar atrás la idea de que este mundo implica posibilidades para otras personas. 
Al respecto, Schutz afirma: "No puedo comprender una cosa social sin reducirla a la actividad humana que la ha creado, y, más allá de ello, sin referir esta actividad humana a los motivos que la originan". Además, en la sociología, desde cuya perspectiva se comprenden los fenómenos sociales, se reitera la necesidad de apoyarse en otras ciencias. Para el caso del emprendimiento se acudirá, entre otras, a la antropología filosófica, que considera al hombre dentro del mundo desde el punto de vista natural y socio-cultural. Así explica Schutz la naturaleza de esta realidad: "La índole biológica del hombre está en la base de su sistema de necesidades y de su orden jerárquico, mientras que su índole espiritual determina las formas de su conocimiento y ambas son el cimiento de la realidad social y cultural" [22].

Es decir, la fenomenología parte de la noción fundamental de sujeto para comprender su realidad y su sentido común, toda vez que cada individuo integra un mundo particular en el cual no solo hay objetos sino otros semejantes, los alter ego, como los denomina Schutz, los cuales actúan en circunstancias típicamente similares y constituyen su acervo de conocimiento [22].

Actualmente, no se cuenta con claridad respecto a lo que representa el emprendimiento social. "El crecimiento del emprendimiento social en términos globales en la última década ha sido impresionante, pero el significado preciso del término permanece como un asunto de debate". Parte de la confusión, en relación con este tipo de actividad emprendedora, radica en el desarrollo teórico dentro de diferentes dominios o áreas de conocimiento, lo cual lleva a una falta de coherencia en el enfoque con el cual es abordada esta temática [23].

Un elemento común en diferentes defi- niciones de emprendimiento social es la búsqueda de soluciones a problemas sociales. El emprendedor social identifica oportunidades que se presentan a sí mismas como problemas que requieren soluciones y se esfuerza por crear emprendimientos para resolverlos. Los emprendedores sociales buscan soluciones para problemas tales como el analfabetismo, la drogadicción o la contaminación ambiental. "Es importante resaltar que el emprendimiento social no es lo mismo que caridad o benevolencia; incluso no es necesariamente sin ánimo de lucro. En esencia, es una actitud benevolente motivada por una necesidad profundamente arraigada de dar a otros, pero va más allá de esto [...] los emprendedores sociales son gente de negocios".

Martin y Osberg [24] buscan diferenciar el emprendimiento social de la provisión de servicios sociales y activismo social. En el caso de la provisión de servicios sociales, individuos comprometidos identifican un equilibrio estable infortunado y desarrollan un programa para atacar esta situación. Sin embargo, "su impacto permanece restringido, su servicio confinado a la población local y su alcance es determinado por los recursos que están en capacidad de atraer. Estas acciones son extremadamente vulnerables, lo que puede llevar a la interrupción o pérdida del servicio por parte de las poblaciones que sirven".

En el caso del activismo social, la motivación sigue siendo la identificación de un equilibrio estable infortunado, pero el actor social se diferencia por su orientación en la acción. "Antes que tomar una acción directa, como lo haría el emprendedor social, el activista social intenta crear cambio a través de la acción indirecta, influenciando a los demás a tomar acción: gobiernos, organizaciones no gubernamentales, con- 
sumidores, trabajadores, entre otros" [24]. La creación de valor social sostenible es la característica clave que diferencia el emprendimiento social de las obras de beneficencia o las actuaciones caritativas de individuos bien intencionados. Los emprendedores sociales "actúan como 'agentes de cambio' en el sector social, innovando y actuando de acuerdo con el deseo de crear un valor social sostenible". Según Reis y Clohesy "el emprendimiento social es fuertemente influenciado por el deseo del cambio social y de sostenibilidad de la organización y los servicios sociales que presta". Es por ello que los planes de negocios de los emprendimientos sociales están centrados especialmente en la sostenibilidad financiera. Así, no se puede hablar de emprendimiento social dejando de lado la sostenibilidad.

Para Roberts y Woods "el emprendimiento social es la construcción, evaluación y persecución de oportunidades para el cambio social transformativo llevado a cabo por individuos visionarios, apasionadamente dedicados". Esta definición permite resaltar otros aspectos claves del emprendimiento social. El primero de ellos es que las oportunidades sociales no se descubren, sino que se construyen gracias a la generación de ideas, se evalúan y, si es el caso, se desarrollan con el ánimo de atender problemas sociales específicos. El segundo aspecto está relacionado con las características de los emprendedores sociales. Es muy importante considerar que, para efectos de una definición de la actividad emprendedora social, no es necesario tener en cuenta las características del individuo. Por lo tanto, se excluyen aportes como los realizados por Hemingway, quien presenta una discusión teórica sobre cómo los valores determinan la propensión del individuo a generar emprendimientos sociales. En este aparte se pretende definir características de la actividad emprendedora social y no del emprendedor social.

Es importante reconocer que el emprendimiento social puede desarrollarse en diferentes sectores. Los autores [25] definen emprendimiento social como "una actividad innovadora, de creación de valor social, que ocurre al interior y a través de los sectores sin ánimo de lucro, de negocios y gubernamental". Lo anterior permite comprender que el emprendimiento social no está aislado del emprendimiento comercial, sino que coexisten.

Note que la distinción entre emprendimientos social y comercial no es dicotómica, sino más acertadamente conceptualizada como un continuo que varía desde lo puramente social a lo puramente económico. Aun en los extremos, sin embargo, existen elementos en común entre ambos. Así, la actividad caritativa debe reflejar realidades económicas, mientras que la actividad económica debe generar valor social [25].

Finalmente, para llegar a una definición propia, que en lugar de constituir una más entre las existentes pretende ser ecléctica y aun así precisa, se considera pertinente revisar la definición propuesta por Martin y Osberg [24]. Para ellos, el emprendimiento social sigue tres componentes:

(1) La identificación de un equilibrio estable pero intrínsecamente injusto que causa exclusión, marginalización o sufrimiento a un segmento de la humanidad que carece de medios financieros o influencia política para alcanzar un beneficio transformador por su cuenta; (2) la identificación de una oportunidad en este equilibrio injusto, desarrollando una proposición de valor social y llevando a utilizar inspiración, creatividad, acción directa, coraje y fortaleza, desafiando de ese modo la hegemonía del estado estable; 
y (3) la creación de un equilibrio estable y nuevo que libera el potencial coartado o alivia el sufrimiento del grupo objetivo, y a través de la imitación y la creación de un ecosistema estable alrededor del nuevo equilibrio, asegura un mejor futuro para el grupo objetivo y la sociedad como un todo [24] .

Es muy importante reconocer que el emprendimiento social busca soluciones para problemas sociales a través de la construcción, evaluación y persecución de oportunidades que permitan la generación de valor social sostenible, alcanzando equilibrios nuevos y estables en relación con las condiciones sociales, a través de la acción directa llevada a cabo por organizaciones sin ánimo de lucro, empresas $u$ organismos gubernamentales.

El emprendimiento social orientado a logros medibles en materia económica y social, se trata de una vía de acción alternativa, inspirada en la lógica empresarial y metódicamente formulada con propósitos sociales, la cual busca canalizar aportes coordinados de distintos actores públicos y privados hacia metas específicas de oportunidad y bienestar.

Las alianzas entre distintos actores sociales garantizan la viabilidad de los programas o proyectos de desarrollo. En esta dirección, se suman fortalezas, se eliminan o neutralizan los puntos débiles y se comparten responsabilidades. Estas estrategias son especialmente exitosas en ámbitos que presentan significativos rezagos, en los cuales se ha venido postergando la ejecución de políticas de desarrollo de corte integral.

El emprendimiento social se apoya en la innovación y la competitividad, instrumentos favorecedores de proyectos y empresas generadoras de empleo y de oportunidades de ingreso sostenible. Por ello, se parte de una perspectiva integral, en la cual es determinante la inversión paralela en tres áreas estratégicas: capital social, que implica el desarrollo de capacidades con el propósito fundamental de propiciar el paso de receptores pasivos a protagonistas activos de su propio desarrollo; obras de infraestructura social, que aseguren el acceso a servicios básicos; y generación de ingresos, lo cual conlleva calificación para el trabajo y proyectos productivos sostenibles. Lo anterior se puede observar en la Figura 5.

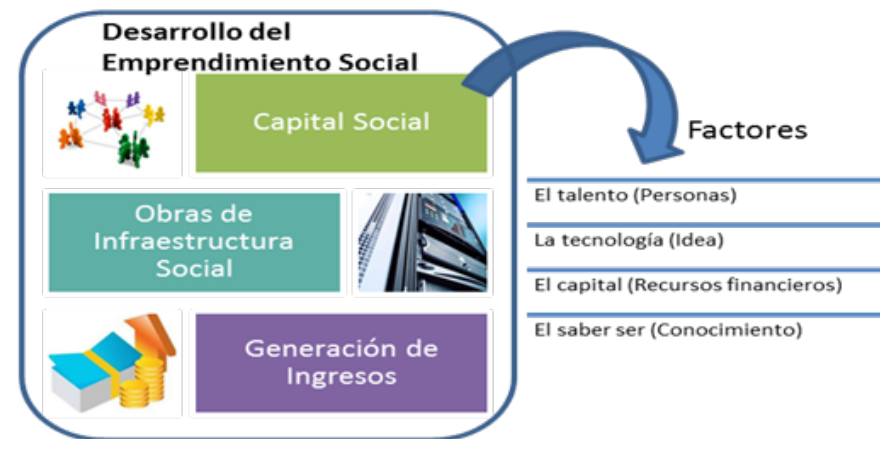

\section{Figura 5. Desarrollo del Emprendimiento Social. Fuente: Elaboración propia de la autora $[25,26]$.}

El emprendimiento social conlleva también metodologías participativas y de fiscalización ciudadana, mecanismos necesarios para la promoción y control social. El aporte solidario de recursos humanos y financieros es la clave para dar contenido económico a los proyectos y asegurar su sostenibilidad. La participación de las instituciones gubernamentales, de los municipios, de los grupos organizados de la sociedad civil, de organismos no gubernamentales, de entes cooperantes y del sector empresarial garantiza la concreción y sostenibilidad de obras y servicios.

\section{Alianza Estratégica}

Si bien es sabido que el objetivo de las redes es compartir información, según Gulati [27] podemos diferenciar a las alian- 
zas, pues para estas el objetivo es tomar acción para hacer algo y hacerlo mejor al trabajar juntos [28]. Además, es importante decir que contar con los alcances estratégicos es conocer el desempeño y su rendimiento en su comportamiento empresarial y observar su entorno.

Es importante conocer el enfoque empresarial. Una estrategia es un plan de acción que define cómo una organización usará sus recursos (tangibles e intangibles) para lograr una mayor ventaja competitiva en el medio ambiente de negocios en el que se desarrolla.

A partir de la revisión de la literatura podemos decir:

Una alianza estratégica es un acuerdo explícito y a largo plazo entre dos o más empresas para desarrollar en común, y gracias a una contribución equitativa de cada uno, una actividad concreta. Un acuerdo que mediante la creación de sinergias permite a cada uno lograr algún(os) objetivo(s) que sería(n) inalcanzable(s) con el esfuerzo individual [29].

Alianzas estratégicas son esencialmente intercambios diádicos por relación de trabajo estrecha entre individuos y organizaciones que consienten en trabajar juntos para un propósito específico; porque pueden lograr más juntos que separados.

El plan que integra las principales metas y políticas de una organización y, a la vez, establece la secuencia coherente de las acciones a realizar. "Una estrategia adecuadamente formulada ayuda a poner orden y asignar, con base tanto en sus atributos como en sus deficiencias internas, los recursos de una organización, con el fin de lograr una situación viable y original, así como anticipar los posibles cambios en el entorno y las acciones imprevistas de los oponentes inteligentes".

$\square \quad$ Las empresas están comenzando a aprender lo que los países siempre han sabido: en un mundo complejo e incierto, lleno de contrincantes peligrosos, es conveniente no atacar solo. Los directivos han sido muy lentos para experimentar con alianzas auténticamente estratégicas. Claro está que ha habido una empresa en participación con otra, también las relaciones contractuales a largo plazo, pero pocas veces se han establecido antes. Una alianza real compromete la independencia fundamental de los actores económicos, a los directivos no les gusta eso. Después de todo, para ellos, la administración ha adquirido el significado de un control total. Las alianzas significan compartir el control. Uno excluye al otro. La globalización impone alianzas, las hace verdaderamente esenciales para la estrategia.

Dentro de los elementos de impacto de las alianzas estratégicas está construir ventaja competitiva para crear cooperación, ser asociativa y abierta a aportar unión y desempeñarse por medio de desembolsos de recursos y conocimientos para soportar la diferencia cultural que existe entre los aliados (ver Figura 6). 


\section{ESTRATEGIAS DE ALIANZAS}

Figura 6. Estrategias de alianzas.

Fuente: elaboración de la autora.

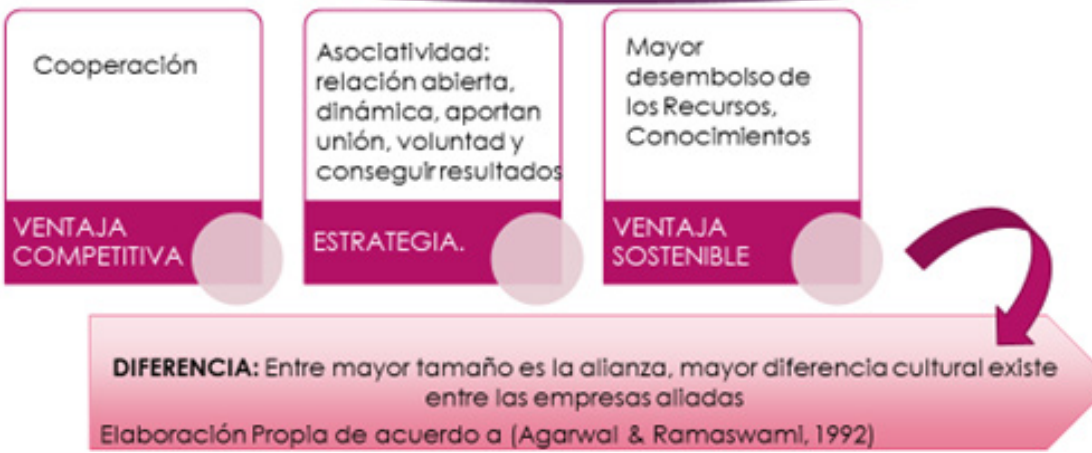

Las alianzas estratégicas son, hoy en día, una modalidad a la que recurren cada vez más empresas para compartir recursos: desarrollados y abundantes en una y escasos en otra; tanto en el aspecto fi122 nanciero y de personal como en las áreas comercial, técnica, tecnológica, fondos, inversiones, credibilidad, prestigio y sistemas establecidos de distribución a nivel nacional e internacional.

Esta manera de operar está adquiriendo gran importancia en el mundo de los negocios, ya que la competencia global se intensifica en relación al acceso a mercados, nuevos productos, tecnología, recursos financieros, costos de fabricación o restricciones ecológicas.

Porter comenta que, ante la apertura comercial, el mundo empresarial ha experimentado cuantiosos cambios. Por un lado, la competencia a nivel mundial eleva los estándares de calidad, innovación, productividad y valor para el consumidor claves del éxito comercial-; y por otro, el campo de acción individual se ve restringido para las empresas.

El elemento importante para lograr la competitividad global son las alianzas o asociaciones estratégicas. El mayor grado de interdependencia ha enseñado a las empresas nacionales que en un mundo cada vez más complejo e incierto, resulta mejor contar con aliados. Las alianzas estratégicas son herramientas indispensables en el arsenal de todo empresario, especialmente en un mundo en el que la globalización avanza cada vez con mayor rapidez.

Las reestructuraciones en la empresa, en general, mediante el reciente recurso de las alianzas estratégicas que se celebran entre firmas de todos tamaños; brinda resultados favorables para algunas de ellas, pues representa un medio para formar empresas más eficientes y competitivas.

Dado que existen una infinidad de formas y posibilidades distintas, se define "alianza estratégica" por la posesión simultánea de estas tres condiciones necesarias y suficientes:

(1) Las firmas que se unen, para conseguir un conjunto de objetivos establecidos previamente, permanecen independientes luego de realizada la alianza.

(2) Las empresas comparten los beneficios de la alianza y controlan determinadas tareas asignadas (esta es la característica que las hace tan difíciles de administrar). (3) Las empresas aliadas contribuyen continuamente en algún área estratégica.

Este concepto no incluye al franchising ni las fusiones, adquisiciones, licencias y 
takeovers. En el cuadro siguiente (Figura 7) se observa el rango de vínculos interempresarios, identificándose cuáles de ellos responden al concepto de "alianza estratégica".
Figura 7. Alianza estratégica entre empresas.

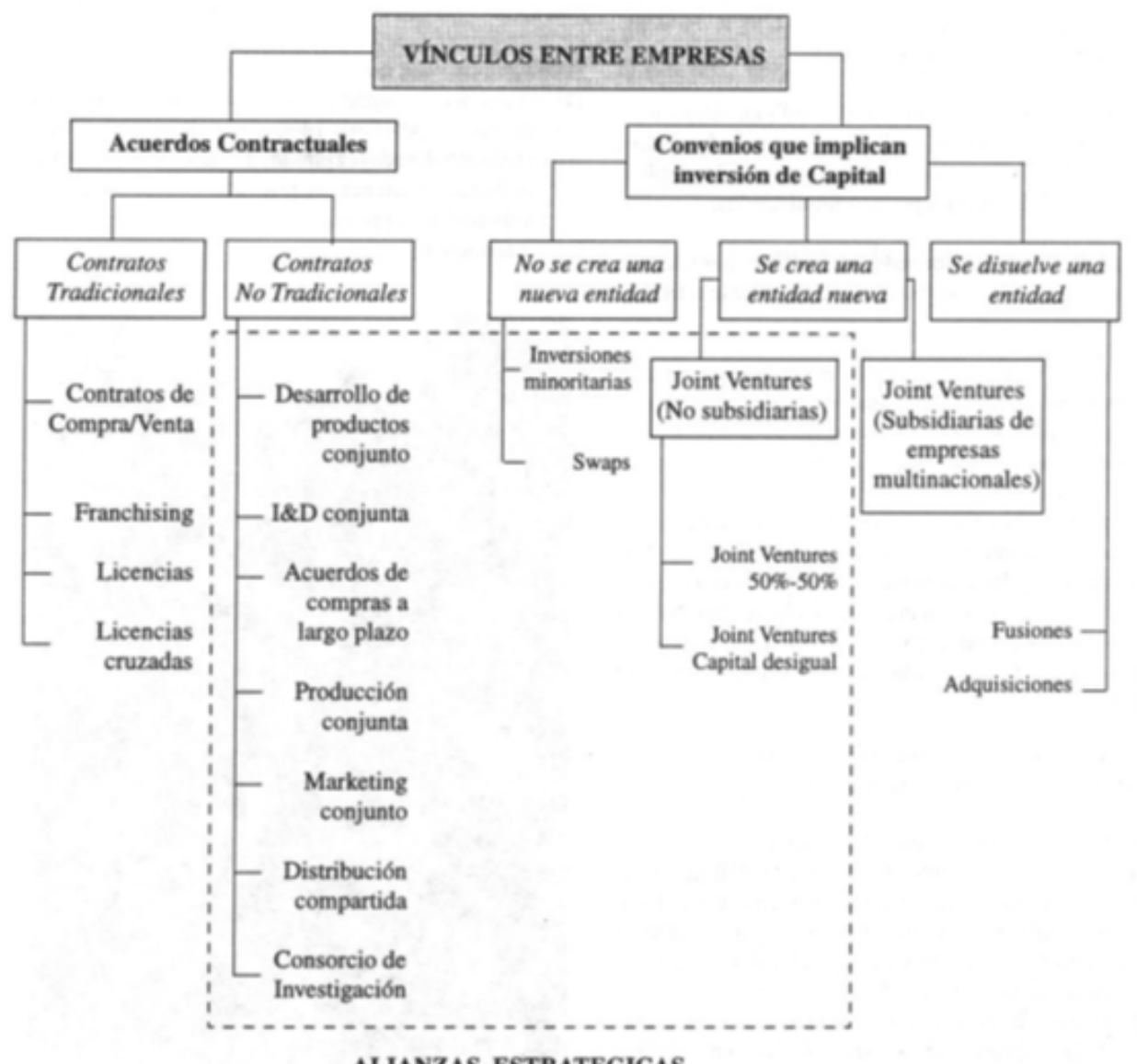

A modo de conclusión, se puede decir que: "Las alianzas constituyen una gran parte del juego de la globalización... Son críticas para ganar con una base global... El modo menos atractivo de tratar de ganar globalmente es pensar que usted solo puede con todo el mundo". Jack Welch, CEO de General Electric. Discurso en Harvard Business School, Octubre 28 de 1987. 


\section{Conclusión}

L

a importancia del emprendimien-

to social está en el impacto sobre

el desarrollo socioeconómico y es especialmente oportuno en países como Colombia, donde la construcción social participativa y solidaria complementa la acción realizada por el Estado frente a los problemas de la sociedad. Por lo tanto, el emprendimiento social se concibe como una propuesta de política pública que de la mano con una estrategia de competitividad aportará un valor agregado para construir sociedad y país.

Es necesario conocer el impacto del concepto de competitividad, comprender todos los aspectos que este involucra y orientar los esfuerzos a la mejora del desempeño competitivo de las organizaciones. El análisis de la competitividad empresarial comprende tres tipos principales de elementos: factores internos, factores externos e índices. Lo anterior nos lleva a contextualizar y a posicionar a las empresas, y proveen los elementos necesarios para realizar análisis orientados a mejorar el desempeño de las empresas exportadoras. Por su parte, el modelo conceptual propuesto de interrelaciones de competitividad provee otra perspectiva desde la cual se pueden analizar las variables que le dan forma al desempeño competitivo de las empresas. Realizar el análisis de los diferentes conceptos nos lleva a crear unas estrategias de direccionamiento estratégico y de gestión administrativa competitiva capaces de dar solución a las necesidades de los actores de la empresa con pocos recursos, puesto que a través de las alianzas lograremos mayor efectividad y reactivar la dinámica de los negocios con velocidades del cambio.

Conocer las estrategias del negocio ge- nera un impulso, ya que se aprovecha el conocimiento que tiene la empresa en un determinado nicho de mercado para contribuir a productos y servicios.

Además, para que una alianza sea exitosa debe existir un equilibrio de fuerzas entre las empresas aliadas con respecto a su temática común; para tal fin las alianzas son excelentes porque proveen, a los empresarios y pequeños negocios, ideas, recursos, herramientas o soluciones que les ayudan a conseguir:

- Costes más bajos (más ganancias).

- Ingresos más altos (de clientes nuevos y actuales).

- Y más tiempo (porque ganan eficiencia). En un entorno cada vez más complejo, cambiante y supra competitivo, la puesta en marcha de una alianza estratégica debe ser una parte importante del repertorio de todo buen gerente o estratega desde el direccionamiento organizacional. Es preciso que, como emprendedor o PYME, comprenda los grandes beneficios de trabajar en alianzas.

Una empresa es competitiva por ser capaz de ofrecer continuamente productos y servicios con atributos valorados por sus clientes. Los mercados cambian, las exigencias de los consumidores también y es clave que la empresa se adapte permanentemente a los cambios a fin de mantener o mejorar sus niveles de competitividad.

La investigación en emprendimiento debe apalancarse con los temas de competitividad y estrategia para mirar los enfoques económicos capaces de crear proyectos innovadores exitosos conforme a los esquemas planteados por [27, 29]. Además, debemos dejar abiertos los siguientes temas claves:

Entender la naturaleza, importancia y evolución deseada de cada alianza.

$\square \quad$ La protección y el incremento de las "core competencies" de la firma. 
Cambiar de paradigma con respecto a los modelos mentales de los gerentes que son designados para trabajar en alianzas.

Identificar los recursos que se van a negociar para no caer en cuellos de botella.

Saber manejar el potencial de evolución de una red de alianzas y la extraordinaria complejidad que significa su coordinación.

\section{Referencias Bibliográfico}

1. M. E. Porter, M. Delgado, C. Ketels, \& S. Stern, Competitive strategy: Techniques for analyzing industries and competitors, 2008.

2. H. Gebauer, A. Gustafsson, \& L. Witell, "Competitive advantage through service differentiation by manufacturing companies", Journal of Business Research, vol. 64, pp. 1270-1280, 2011.

3. U. Orozco-Rosas, E. Ahumada-Tello, \& R. Zárate, "Factores en el desarrollo de la competitividad: El modelo de competitividad sistemática en la industria del software en Baja California", Global Conference on Business and Finance Proceedings, vol. 5, pp. 1236-1245, 2010.

4. E. H. Mora-riapira, \& M. Vera-colina, "Planificación estratégica y niveles de competitividad de las Mipymes del sector comercio en Bogotá", Estudios Gerenciales, vol. 31, 2015.

5. G. Dosi, "Sources, procedures, and microeconomic effects of innovation", Journal of economic literature, pp. 1120-1171, 1988.

6. R. Horta, \& A. Jung, “Competitividad e industria manufacturera. Aportes para un marco de análisis", Revista electrónica de la Facultad de Ciencias Económicas de la Universidad Católica, vol. 1, pp. 1-38, 2002. 7. I. Barquero, "El estado y la competitivi- dad de la micro, pequeña y mediana empresa". PNUD, 2003.

8. P. Krugman, "The fall and rise of development economics", Rethinking the development experience, pp. 39-59, 1994.

9. J. B. Valencia, A. I. Z. Torres, \& C. F. O. Paniagua, "Variables e Índices de Competitividad de las Empresas Exportadoras, utilizando el PLS".Cimexus, vol. 10, pp. 13-32, 2016.

10. M. E. Porter, E. B. Campos, C. M. Moreno., \& M. P. S. Sánchez, Ventaja competitiva: creación y sostenibilidad de un rendimiento superior, 2010.

11. M. D. P. Buitrago Velandia, Teoría de la estrategia y la competitividad: estado del arte desde la perspectiva de Michael E. Porter y su aplicación en Colombia, 2010. 12. A. D. Chandler, T. Hikino., \& A. D. Chandler, Scale and scope: The dynamics of industrial capitalism. Harvard University Press, 2009.

13. H. I. Ansoff, \& A. Z. Sanvicente, Estrategia empresarial, 1977.

14. R. Z. Villegas, "Olas paradigmáticas de la estrategia: enfoque hermenéutico", Revista Ciencias Estratégicas, vol. 15, pp. 83-92, 2007.

15. A. Tilman, W. Hillebrand \& J. Meyer-Stamer, "Building Systemic Competitiveness. Concept and Case Studies from Mexico, Brazil, Paraguay, Korea and Thailand", Reports and Working Papers of the German Development Institute, 1998.

16. J. Lombana, Competitiveness and trade policy problems in agricultural exports: A perspective of producing/exporting countries in the case of Banana Trade to The European Union. Saarbrucken, Alemania: Müller Verlag, 2006.

17. R. Cantillón, Essai Sur la Nature du Commerce en General (H. Higgs, Ed. y Trans.). Londrés, UK: Macmillan. (Traducción publicada en 1931), 1755.

18. S. Shane., y S. Venkataraman, "The 
Promise of Entrepreneurship as a Field of Research", Academy of Management Review, vol. 25, pp. 217-226, 2010.

19. M. Casson, The Entrepreneur. An Economic Theory. Oxford, Reino Unido: Martin Robertson \& Company, 1982.

20. Cecodes, Acerca de Cecodes, 2012. Disponible en: http://www.cecodes.org.co/ index.php/acerca-de-cecodes.html

21. C. I. Orrego Correa, "La fenomenología y el emprendimiento", Pensamiento \& Gestión, vol. 27, pp. 235-252, 2009.

22. A. Schutz, Estudios sobre la teoría social. Escritos II. Buenos Aires: Amorrortu, 2003.

23. J. Weerawardena., y G. Sullivan, “Investigating social entrepreneurship: A multidimensional model", Journal of World Business, vol.41, pp. 21-35, 2006.

24. R. L. Martin, \& S. Osberg, "Social entrepreneurship: The case for definition", Stanford social innovation review, vol. 5, pp. 28-39, 2007.

25. J. Austin, H. Stevenson, y J. Wei-Skillern, "Social and Commercial Entrepreneurship: Same, Different, or Both?", Entrepreneurship: Theory \& Practice, vol. 30, pp. 1-22, 2006.

26. E. Chell, Social Enterprise and Entrepreneurship. International Small Business, 2007.

27. R. Gulati, "Alliances and Networks", Strategic Management Journal, vol. 19, 1998.

28. E. Laumann, Interlocking and Radial Friendship Networks: A Cross- Sectorial Analysis. Mimeographed. Ann Arbor: University of Michigan, 1968.

29. M. Menguzzato Boulard, La triple lógica de las alianzas estratégicas, en Dirección de empresas de los noventa. Madrid: Editorial Civitas, 1995. 


\section{Este artículo se cita:}

Citación estilo

H. Hernandez, "Factores críticos para promover la calidad en el sector salud del Departamento del Atlántico", Investigación e Innovación en Ingenierias, vol. $4, \mathrm{n}^{\circ} .2, \mathrm{pp} . \mathrm{Xx}-\mathrm{xx}$, 2016. 